\title{
Different Influences on Tacrolimus Pharmacokinetics by Coadministrations of Zhi Ke and Zhi Shi in Rats
}

\author{
Shiuan-Pey Lin, ${ }^{1}$ Ping-Ping Wu, ${ }^{1}$ Yu-Chi Hou, ${ }^{1}$ Shang-Yuan Tsai, ${ }^{1}$ Meng-Ju Wang, ${ }^{2}$ \\ Shih-Hua Fang, ${ }^{3}$ and Pei-Dawn Lee Chao ${ }^{1}$ \\ ${ }^{1}$ School of Pharmacy, China Medical University, Taichung 40402, Taiwan \\ ${ }^{2}$ School of Chinese Pharmaceutical Sciences and Chinese Medicine Resources, China Medical University, Taichung 40402, Taiwan \\ ${ }^{3}$ Institute of Athletics, National Taiwan College of Physical Education, Taichung 40404, Taiwan
}

Correspondence should be addressed to Pei-Dawn Lee Chao, pdlchao@gmail.com

Received 12 July 2010; Revised 19 October 2010; Accepted 28 December 2010

Copyright (๑) 2011 Shiuan-Pey Lin et al. This is an open access article distributed under the Creative Commons Attribution License, which permits unrestricted use, distribution, and reproduction in any medium, provided the original work is properly cited.

Tacrolimus, an immunosuppressant with narrow therapeutic window, has been used widely in transplant patients. Grapefruit juice and pomelo have been reported to increase the blood levels of tacrolimus. Zhi Ke and Zhi Shi, the ripe peels and unripe fruits of Citrus aurantium which is chemotaxonomically related to grapefruit and pomelo, are in wide use in clinical Chinese medicine. To investigate the possible interaction of these two Citrus herbs with tacrolimus, male Sprague-Dawley rats were orally given tacrolimus $(1.5 \mathrm{mg} / \mathrm{kg})$ with and without Zhi Ke and Zhi Shi decoctions in a cross-over design. Blood samples were withdrawn via cardiopuncture at specific time and quantitated by a microparticle enzyme immunoassay. In addition, to explore the mechanism of interaction, LS 180 cell line was used for the transport study of rhodamine 123, a typical substrate of P-glycoprotein (P-gp). The results showed that Zhi Shi significantly decreased the $C_{\max }$ and $\mathrm{AUC}_{0-t}$ of tacrolimus by $72.4 \%$ and $72.0 \%$, respectively, whereas Zhi Ke did not affect tacrolimus pharmacokinetics. LS 180 cell line study indicated that Zhi Shi increased the efflux activity of P-gp, enabling us to explain the decreased oral bioavailability of tacrolimus caused by Zhi Shi. Hence, we suggest that Zhi Shi be contraindicated for transplant patients treated with tacrolimus to reduce the risk of allograft rejection.

\section{Introduction}

Zhi Ke and Zhi Shi, the dried ripe peels and unripe fruits of Citrus aurantium, respectively, are widely used in clinical Chinese medicine. The ripe fruits were harvested in July and the unripe ones were harvested earlier in May-June. Zhi Ke was clinically used in easing pain of epigastrium or abdomen and poor appetite due to stagnation of spleen and stomach [1]. The constituents in Zhi Ke include naringin, naringenin, hesperidin, neohesperidin, $\beta$-carotene, l-carotene, citraurin, synephrine, N-methyltyramine, auraptenol, aurantiamaric acid, aurantiamarin, and so forth $[2,3]$. In contrast, Zhi Shi was often prescribed for gastrointestinal diseases such as food retention, constipation, distention of chest, and epigastrium [1]. The constituents in Zhi Shi include naringin, naringenin, hesperidin, neohesperidin, $\beta$-carotene, 1 -carotene, citraurin, synephrine, N-methyltyramine, auraptenol, rhoifolin, lonicerin, poncirin, limonin, 5-O-desmethylnobiletin, and so forth $[2,3]$.

Tacrolimus, a lipophilic macrolide isolated from Streptomyces tsukubaensis, is an important immunosuppressant widely used in transplant patients, but with narrow therapeutic window. The half-life of tacrolimus in human is 8.7$11.3 \mathrm{~h} \mathrm{[4].} \mathrm{The} \mathrm{adverse} \mathrm{effects} \mathrm{of} \mathrm{tacrolimus} \mathrm{included} \mathrm{neu-}$ rotoxicity, nephrotoxicity, gastrointestinal toxicity, hyperkalaemia, hypertension, and myocardial hypertrophy [5-8]. On the contrary, subtherapeutic level of tacrolimus may result in acute rejection of xenografts $[9,10]$. Therefore, whatever significantly affecting the absorption or disposition of tacrolimus is of clinical importance. Tacrolimus is known as a substrate of P-glycoprotein (P-gp), a multidrug efflux transporter, and cytochrome P450 3A4 (CYP3A4) [11-13]. Consequently, any modulator of P-gp or CYP3A4 may alter the pharmacokinetics of tacrolimus.

In recent decades, several clinical studies have found that grapefruit juice and pomelo significantly increased the blood levels of tacrolimus [14-18]. The fruits of Citrus aurantium share many common constituents with grapefruit and pomelo, such as naringin, naringenin, hesperidin, and so forth [3]. Therefore, we suspect that Zhi Ke and Zhi Shi may be subject to relevant interaction with tacrolimus as grapefruit juice and pomelo did [15-18]. Our previous 
study has reported that Zhi Ke significantly increased the oral bioavailability and toxicity of cyclosporine in pigs [19]. Another study found that Zhi Shi significantly decreased the oral bioavailability of cyclosporine in rats [20]. This study in turn investigated the effects of Zhi Ke and Zhi Shi on the pharmacokinetics of tacrolimus in rats. Furthermore, possible mechanism of interaction was explored by using cell line model.

\section{Methods}

2.1. Chemicals. Tacrolimus (Prograf, $5 \mathrm{mg} / \mathrm{mL}$ ) was purchased from Fujisawa Pharmaceutical Company (Osaka, Japan). The crude drugs of Zhi Ke and Zhi Shi were purchased from a Chinese drugstore in Taichung and identified by microscopic examination. The specimens were deposited at the Institute of Chinese Pharmaceutical Sciences. Dimethyl sulfoxide (DMSO), 3- $\left(4^{\prime}, 5^{\prime}\right.$-dimethylthiazol-2'-yl)-2,5-diphenyltetrazolium bromide (MTT), rhodamine 123, sodium dodecyl sulfate (SDS), Triton X-100, and verapamil were obtained from Sigma (St. Louis, MO, USA). 6,7-Dimethoxycoumarin was purchased from Aldrich (Milwaukee, WI, USA). Dulbecco's Modified Eagle Medium (DMEM), trypsin/EDTA, nonessential amino acid, Hank's Buffered Salt Solution (HBSS), and 4-(2-hydroxyethyl)1-piperazineethanesulfonic acid (HEPES) were purchased from Invitrogen (Grand Island, NY, USA). IMx kit was supplied by Abbott Laboratories (Abbott Park, IL, USA). Milli-Q plus water (Millipore, Bedford, MA, USA) was used for all preparations.

2.2. Instrumentation. The HPLC apparatus included a pump (LC-10AS, Shimadzu, Japan) and an UV/VIS detector (SPD10A, Shimadzu, Japan). The RP-18e column (Apollo, $5 \mu \mathrm{m}$, $250 \times 4.6 \mathrm{~mm}$ ) was equipped with a guard column (LiChrospher 100, $5 \mu \mathrm{m}$ ). The mobile phase consisted of acetonitrile and water at a ratio of $22: 78(\mathrm{v} / \mathrm{v})$. The UV detector was set at $280 \mathrm{~nm}$, and the flow rate was $1.0 \mathrm{~mL} / \mathrm{min}$.

\subsection{Preparation and Characterization of Zhi Ke and Zhi Shi} Decoctions. To prepare decoctions, $25 \mathrm{~g}$ of Zhi Ke or Zhi Shi were added to $500 \mathrm{~mL}$ of water, and then heated on a gas stove. After boiling, the mixture was heated gently until the volume of decoction was reduced to less than $100 \mathrm{~mL}$. The mixture was filtered while hot, and sufficient hot water was added to make $100 \mathrm{~mL}$. Characterization of the decoctions was carried out through quantitation of naringin and naringenin by HPLC following a previous method with little modification [19]. Briefly, $3.0 \mathrm{~mL}$ of decoction was mixed with $7.0 \mathrm{~mL}$ of methanol, and centrifuged at $10,000 \mathrm{~g}$ for $15 \mathrm{~min}$. A portion of the supernatant was mixed with equal volume of methanol containing $40.0 \mu \mathrm{g} / \mathrm{mL}$ of 6,7-dimethoxycoumarin as internal standard. After filtered through $0.45 \mu \mathrm{m}$ filter, the sample was subject to HPLC analysis. The remaining decoction was frozen at $-30^{\circ} \mathrm{C}$ for later use.

2.4. Animals and Drug Administration. Male SpragueDawley rats were supplied by National Laboratory Animal
Center (Taipei, Taiwan) and kept at least 1 week under environmentally controlled conditions with free access to food and water before experiment. After overnight fast, six rats aged $8-12$ weaks and weighing $300-400 \mathrm{~g}$ were given $1.5 \mathrm{mg} / \mathrm{kg}$ of tacrolimus orally with and without decoction of Zhi Ke or Zhi Shi $(2 \mathrm{~g} / \mathrm{kg})$ in a cross-over design. Drug administration was carried out via gastric gavage, and the decoctions were given 10 min before tacrolimus. Equal volume of water as the decoction was administered as control vehicle. Food was withheld for another $3 \mathrm{~h}$ after dosing. One-week was allowed for washout between two treatments. All animal experiments adhered to "The Guidebook for the Care and Use of Laboratory Animals (2002)" (Published by the Chinese Society of Animal Science, Taiwan), and the experimental protocol had been reviewed and approved by the Institutional Animal Care and Use Committee of China Medical University, Taiwan.

\subsection{Blood Collection and Determination of Tacrolimus Blood} Concentration. Blood samples $(0.3 \mathrm{~mL})$ were withdrawn via cardiopuncture at $0,5,15,30,60,120,240$, and 480 min after oral dosing of tacrolimus. Blood samples were collected in vacutainer tubes containing EDTA and analyzed within $24 \mathrm{~h}$.

Blood tacrolimus concentration was assayed by a microparticle enzyme immunoassay (MEIA) using IMx kit. All the procedures, calibration curve, and validation followed the working protocols provided by the supplier. The assay was calibrated from 3.0 to $30.0 \mathrm{ng} / \mathrm{mL}$, and the lower limit of detection was $1.5 \mathrm{ng} / \mathrm{mL}$.

2.6. Cell Line. LS 180, the human colon adenocarcinoma cell line, was obtained from the Food Industry Research and Development Institute (Hsinchu, Taiwan). Cells were cultured in DMEM medium supplemented with 10\% fetal bovine serum (Biological Industries Ltd., Kibbutz Beit Haemek, Israel), $0.1 \mathrm{mM}$ nonessential amino acid, 100 units $/ \mathrm{mL}$ of penicillin, $100 \mu \mathrm{g} / \mathrm{mL}$ of streptomycin, and $292 \mu \mathrm{g} / \mathrm{mL}$ of glutamine. Cells were grown at $37^{\circ} \mathrm{C}$ in a humidified incubator containing $5 \% \mathrm{CO}_{2}$. The medium was changed every other day, and cells were subcultured when $80 \%$ to $90 \%$ confluency was reached.

2.7. Cell Viability Assay. The effects of Zhi Shi decoction, verapamil, and DMSO on the viability of LS 180 cells was evaluated by MTT assay [21]. Cells were seeded into a 96well plate. After overnight incubation, the tested agents were added into the wells and incubated for $72 \mathrm{~h}$, then $10 \mu \mathrm{L}$ of MTT $(5 \mathrm{mg} / \mathrm{mL})$ was added into each well and incubated for additional $4 \mathrm{~h}$. During this period, MTT was reduced to formazan crystal by live cells. Acid-SDS (10\%) solution was added to dissolve the purple crystal at the end of incubation, and the optical density was detected at $570 \mathrm{~nm}$ by a microplate reader (BioTex, Highland Park, Winooski, VT, USA).

2.8. Transport Assay. The transport assay of rhodamine 123 was modified from a previous method [22]. Briefly, LS 180 cells $\left(1 \times 10^{5}\right)$ were cultured in each well in a 96-well plate. 
TABLE 1: Pharmacokinetic parameters of tacrolimus in six rats given tacrolimus $(1.5 \mathrm{mg} / \mathrm{kg}$ ) alone and coadministered with $2 \mathrm{~g} / \mathrm{kg}$ of Zhi Ke and Zhi Shi decoctions.

\begin{tabular}{lccc}
\hline Parameter & $\begin{array}{c}\text { Treatment } \\
\text { Tacrolimus } \\
\text { alone }\end{array}$ & $\begin{array}{c}\text { Tacrolimus } \\
\text { Zhi Ke }\end{array}$ & $\begin{array}{c}\text { Tacrolimus }+ \\
\text { Zhi Shi }\end{array}$ \\
\hline$T_{\max }(\mathrm{min})$ & $22.5 \pm 8.2$ & $27.5 \pm 6.1$ & $25.0 \pm 7.7$ \\
$C_{\max }\left(\mathrm{ng} \cdot \mathrm{mL}^{-1}\right)$ & $19.2 \pm 4.2^{\mathrm{a}}$ & $17.7 \pm 4.8$ & $5.3 \pm 0.8^{\mathrm{b}}$ \\
& & & $(-72.4 \%)$ \\
$\mathrm{AUC} \mathrm{C}_{0-480}$ & $2485.4 \pm 964.2^{\mathrm{a}}$ & $2330.5 \pm 268.8^{\mathrm{a}}$ & $696.2 \pm 182.5^{\mathrm{b}}$ \\
$\left(\mathrm{ng} \cdot \mathrm{min} \cdot \mathrm{mL}^{-1}\right)$ & & & $(-72.0 \%)$ \\
$\mathrm{MRT}(\mathrm{min})$ & $144.3 \pm 15.2$ & $147.6 \pm 9.1$ & $127.0 \pm 29.9$ \\
\hline
\end{tabular}

$T_{\max }$ : time to reach $C_{\max }$.

$C_{\max }$ : the peak blood concentration.

$\mathrm{AUC}_{0-480}$ : area under the blood concentration-time curve to $480 \mathrm{~min}$. MRT: mean residence time.

Data expressed as mean \pm S.D. Means in a row without a common superscript differ, $P<.05$.

After overnight incubation, the medium was removed and washed three times with ice-cold PBS buffer. Rhodamine 123 in HBSS $(10 \mu \mathrm{M}, 100 \mu \mathrm{L})$ was added into each well and incubated at $37^{\circ} \mathrm{C}$. After 1 -h incubation, the supernatants were removed and washed for three times with ice-cold PBS. Then, Zhi Shi decoction, verapamil (as positive control) and DMSO were added to correspondent wells and incubated at $37^{\circ} \mathrm{C}$. After 4 -h incubation, the medium was removed and the cells were washed three times with ice-cold PBS. Subsequently, $100 \mu \mathrm{L}$ of $0.1 \%$ Triton X-100 was added to lyse the cells and the fluorescence was measured with excitation at $485 \mathrm{~nm}$ and emission at $528 \mathrm{~nm}$. To quantitate the content of protein in each well, $10 \mu \mathrm{L}$ of cell lysate was added to $200 \mu \mathrm{L}$ of diluted protein assay reagent (Bio-Rad, Hercules, CA, USA) and the optical density was measured at $570 \mathrm{~nm}$. The relative intracellular accumulation of rhodamine 123 was calculated by comparing with that of control.

2.9. Data Analysis. Noncompartment model of WinNonlin (version 1.1, SCI software, Statistical Consulting Inc., Apex, NC, USA) was used for the computation of pharmacokinetic parameters of tacrolimus. The area under the blood concentration-time curve $\left(\mathrm{AUC}_{0-t}\right)$ was calculated by the trapezoidal rule to the last point. Pharmacokinetic parameters among various treatment groups were compared using one-way ANOVA with Scheffe's test, taking $P<.05$ as significant.

\section{Results}

Figure 1 is the pictures of crude drugs of Zhi Ke and Zhi Shi. Quantitation results showed that the contents of naringin and naringenin in Zhi Ke decoction were 280. 5 and $5.0 \mu \mathrm{g} / \mathrm{mL}$, respectively, and in Zhi Shi decoction were 164.3 and $5.2 \mu \mathrm{g} / \mathrm{mL}$, respectively.

Figure 2 depicts the mean blood concentration-time profiles of tacrolimus after treatments with tacrolimus alone and coadministered with Zhi Ke and Zhi Shi decoctions in

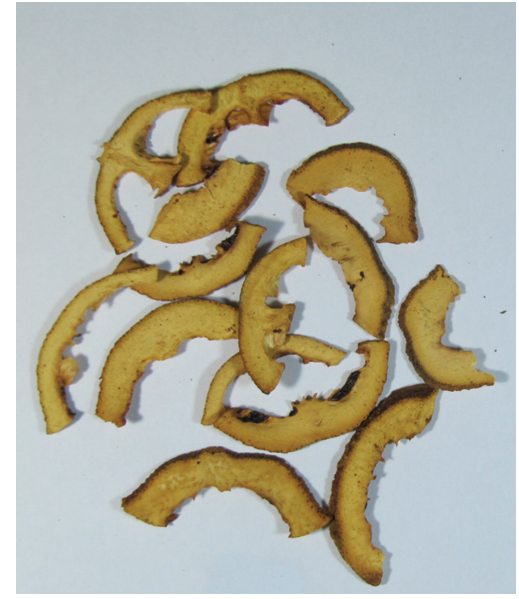

(a)

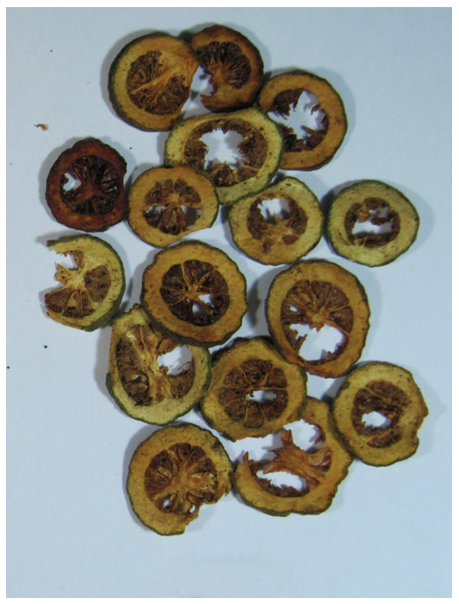

(b)

Figure 1: Crude drugs of Zhi Ke (a) and Zhi Shi (b).

rats. The pharmacokinetic parameters of tacrolimus after various treatments are listed in Table 1. Following coadministration with Zhi Ke, the blood profile of tacrolimus was essentially superposable with that of control. Nonetheless, the blood levels of tacrolimus were markedly decreased since the very early absorption phase upon coadministration with Zhi Shi, which significantly decreased the peak blood concentration $\left(C_{\max }\right)$ and area under the concentration curve $\left(\mathrm{AUC}_{0-t}\right.$ ) of tacrolimus by $72.4 \%$ and $72.0 \%$, respectively. The time to peak concentration $\left(T_{\max }\right)$ and mean residence time (MRT) of tacrolimus were not affected.

To explore the mechanism of Zhi Shi-tacrolimus interaction, LS 180 cell model was used for the transport assay of rhodamine 123. Through MTT assay, Zhi Shi decoction showed no significant influence on cell viability at the tested concentrations. In transport study, the intracellular accumulation of rhodamine 123 measured after 4-h incubation with tested agents is shown in Figure 3. The positive control verapamil $(200 \mu \mathrm{M})$ significantly increased the intracellular accumulation of rhodamine 123 by $46.5 \%$. On the contrary, Zhi Shi significantly reduced the intracellular accumulation 


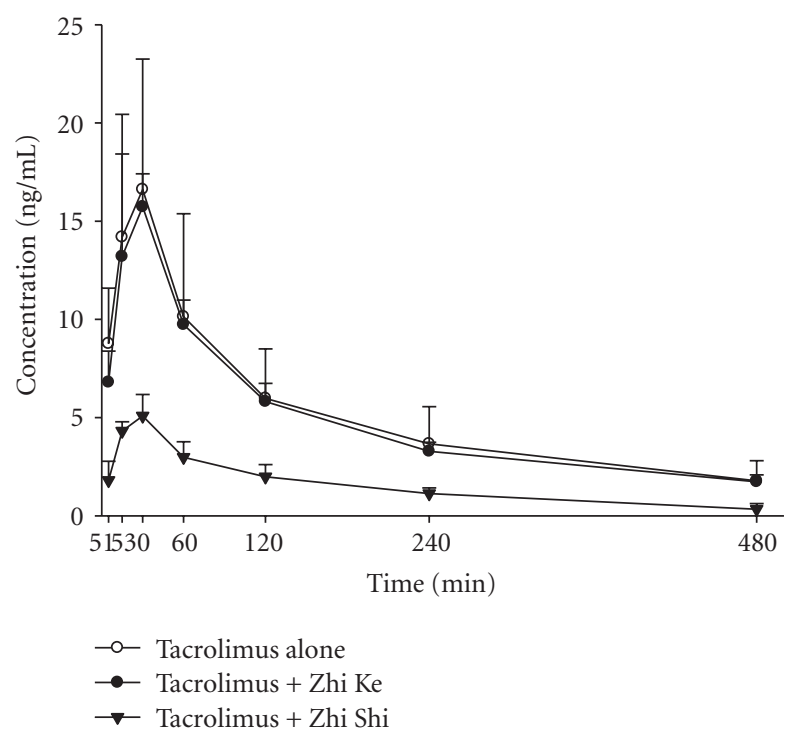

Figure 2: Mean ( \pm S.D.) blood concentration-time profiles of tacrolimus after oral administration of tacrolimus alone $(1.5 \mathrm{mg} / \mathrm{kg})$ $(\circ)$ and coadministrations with $2 \mathrm{~g} / \mathrm{kg}$ of Zhi $\mathrm{Ke}(\bullet)$ and Zhi Shi $(\mathbf{\nabla})$ decoctions.

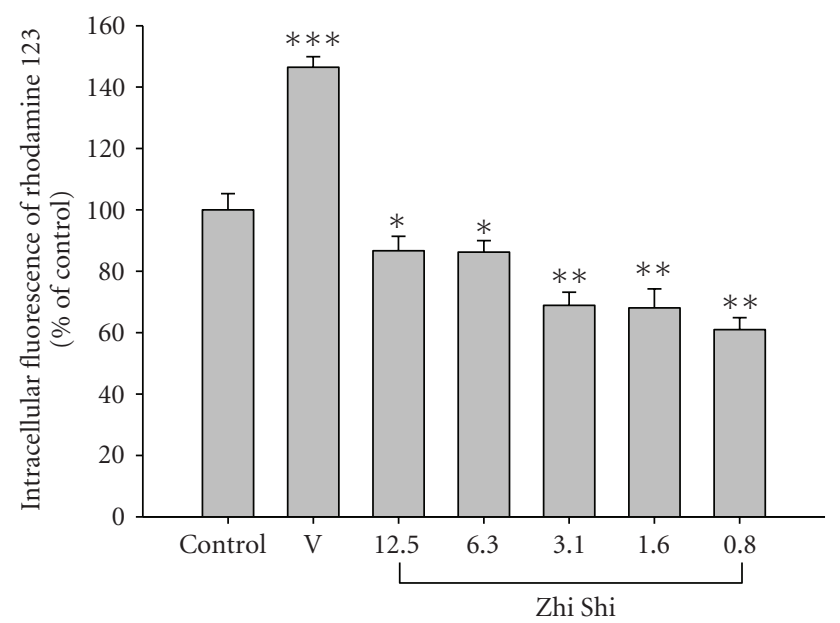

Figure 3: Effects of Zhi Shi $(\mathrm{mg} / \mathrm{mL})$ and verapamil (V, $200 \mu \mathrm{M})$ on the accumulation of rhodamine 123 in LS 180 cells. Data expressed as mean \pm S.D. ${ }^{*} P<.05,{ }^{* *} P<.01,{ }^{* * *} P<.001$.

of rhodamine 123 by $39.0-13.9 \%$ at the concentration range of $0.8-12.5 \mathrm{mg} / \mathrm{mL}$.

\section{Discussions}

In this study, a rat model was employed to evaluate the effects of two Citrus herbs on tacrolimus pharmacokinetics. The markedly decreased $C_{\max }$ and $\mathrm{AUC}_{0-t}$ of tacrolimus caused by coadministration of Zhi Shi indicated that the oral bioavailability of tacrolimus was significantly reduced, whereas Zhi Ke did not alter tacrolimus bioavailability. These results are similar to our previous work reporting the effects of Zhi Ke and Zhi Shi on the pharmacokinetics

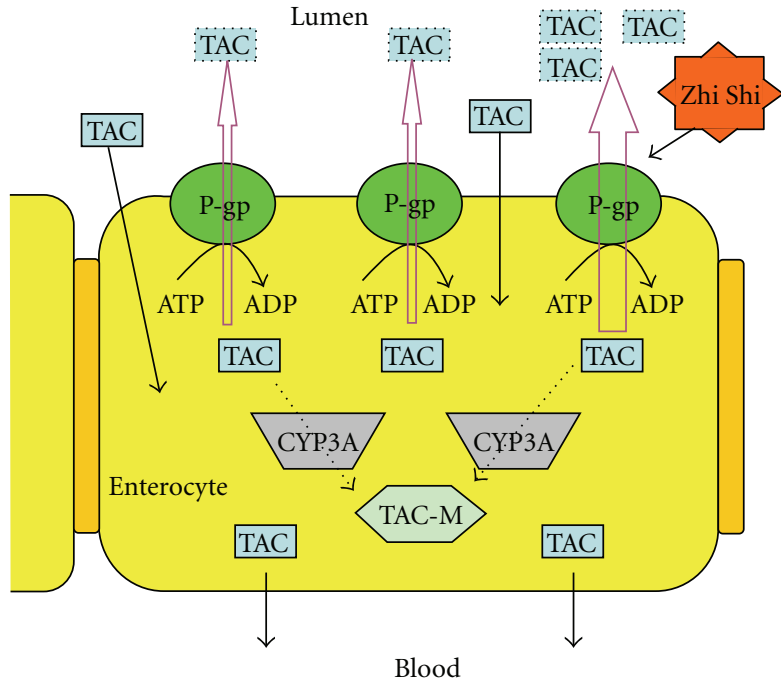

FIgURE 4: Illustration of the inhibition of Zhi Shi on tacrolimus (TAC) absorption through activating the efflux function of P-gp on the cell membrane of enterocytes. TAC-M: TAC metabolites.

of cyclosporine, also a substrate of P-gp and CYP 3A4, in rats [20]. For clinical implication, we strongly suggest that concurrent use of Zhi Shi with cyclosporine or tacrolimus should be avoided to reduce the risk of allograft rejection.

Grapefruit juice and pomelo have been well known in that they could elevate the blood levels of cyclosporine and tacrolimus $[14,15,17,18,23,24]$. So far, many constituents have been identified to be the possible causative agents for the interactions between grapefruit juice and western medicines, which were substrates of P-gp or/and CYP3A4 [25]. Among the putative causative constituents, $6^{\prime}, 7^{\prime}$-dihydroxybergamottin and bergamottin, two minor furanocoumarins, have been demonstrated to inhibit enteric CYP3A4 and P-gp [26-32]. However, most of these conclusions were drawn essentially from in vitro studies. Until recently, a human study has pointed out that lack of interaction with felodipine when furanocoumarin-free grapefruit juice was coadministered [33]. As a result, $6^{\prime}, 7^{\prime}$ dihydroxybergamottin and bergamottin can be thought responsible for the inhibition of intestinal CYP3A4 and Pgp resulting from grapefruit juice consumption. Although Citrus aurantium belongs to the same genus as grapefruit, our HPLC analysis showed no obergamottin in both Zhi Ke and Zhi Shi decoctions. However, detection of $6^{\prime}, 7^{\prime}-$ dihydroxybergamottin in the decoctions was not attempted because of the unavailability of authentic standard. The opposite influences on tacrolimus blood levels between grapefruit juice and Zhi Shi decoction might be in part attributable to the presence of furanocoumarins in grapefruit juice but not in Zhi Shi.

The origins of Zhi Ke and Zhi Shi are from the fruits of the same plant, but harvested at different seasons. The different influences on tacrolimus pharmacokinetics between coadministrations of Zhi Ke and Zhi Shi might be due to the change of constituents during fruit ripening. Through 
comparing the concentrations of naringin and naringenin in two decoctions, the involvement of these two flavanones in the interaction can be excluded. On the other hand, the difference of parts used between Zhi Ke and Zhi Shi might be another possible reason to result in different effect. What constituents in Zhi Shi actually resulted in the marked decrease of blood tacrolimus levels is still on the agenda of our future research.

Because the elimination half-lives of tacrolimus between treatments with tacrolimus alone and coadministration with Zhi Shi were not significantly different, we thus propose that the decreased bioavailability of tacrolimus caused by Zhi Shi should primarily occur at the absorption site. Two mechanisms affecting the fate of tacrolimus at absorption site have been identified: pumped out by P-gp in intestine surface at the first stage and, subsequently, the residuals metabolized by CYP3A4 in intestine and liver [11-13]. Therefore, reduced oral absorption of tacrolimus may be associated with the activation of P-gp and/or CYP3A4.

To explore the association of P-gp with the interaction, transport assay of rhodamine 123 was conducted using LS 180 cells. As shown in Figure 3, contrary to verapamil (a positive control as P-gp inhibitor), Zhi Shi decreased the intracellular accumulation of rhodamine 123, indicating that the efflux function of P-gp was activated. The magnitude of P-gp activation was found not proportional to the concentration of Zhi Shi, which might be accounted for by the complex chemical constituents in Zhi Shi decoction. We contemplate that it is likely that some constituents inhibiting P-gp were coexistent in Zhi Shi decoction and counteracted partial P-gp induction effect at high concentration. Based on this in vitro result, the increased P-gp activity might in part explain the decreased blood levels of tacrolimus in rats as illustrated in Figure 4.

With regard to the effect on CYP3A4, grapefruit juice has been found to decrease intestinal CYP3A4 protein expression $[34,35]$, which may explain the increased blood levels of tacrolimus [36]. On the contrary, the decreased blood levels of tacrolimus caused by Zhi Shi might stem from induction of CYP3A4 activity. However, an in vitro study has revealed that Zhi Shi exerted weak inhibition on CYP3A4 activity in testosterone 6-hydroxylation by human liver microsomes [37], which was apparently not consistent with our in vivo evidence. This in vitro-in vivo discrepancy led us to propose that most constituents in herbal decoctions were hydrophilic and subjected to metabolic transformations in the gut lumen before entering the enterocytes and hepatocytes. How CYP3A4 is involved in herb-drug interaction requires a different approach from drug-drug interaction in western medicines. Therefore, understanding the presystemic metabolism of herbal constituents is essential for the establishment of a rational model more mimicking the biological system.

In conclusion, Zhi Shi significantly decreased the oral bioavailability of tacrolimus in rats, whereas Zhi Ke exerted no influence. We suggest that Zhi Shi be contraindicated for transplant patients treated with tacrolimus to reduce the risk of allograft rejection.

\section{Acknowledgments}

This work was in part supported by the National Science Council, ROC (NSC95-2320-B039-023-MY2 and NSC 962320-B-039-037-MY3), China Medical University (CMU98S-32, CMU98-S-34) and Taiwan Department of Health Cancer Research Center of Excellence (DOH99-TD-C-111005).” S.-P. Lin and P.-P. Wu contributed equally to this work.

\section{References}

[1] Chinese Pharmacopoeia Commission, The Pharmacopoeia of the People's Republic of China, Chemical Industry Press, Beijing, China, 2000.

[2] Y. S. Wang and W. Y. Luo, "Studies on quantitative determination of total flavonoid in qingpi, zhiqiao and zhishi by TLCdensitometric methods," Zhongguo Zhong Yao Za Zhi, vol. 14, no. 4, pp. 230-255, 1989.

[3] F. Pellati, S. Benvenuti, and M. Melegari, "High-performance liquid chromatography methods for the analysis of adrenergic amines and flavanones in Citrus aurantium L. var. amara," Phytochemical Analysis, vol. 15, no. 4, pp. 220-225, 2004.

[4] R. Venkataramanan, A. Jain, E. Cadoff et al., "Pharmacokinetics of FK 506: preclinical and clinical studies," Transplantation Proceedings, vol. 22, no. 1, pp. 52-56, 1990.

[5] R. P. Kershner and W. E. Fitzsimmons, "Relationship of FK506 whole blood concentrations and efficacy and toxicity after liver and kidney transplantation," Transplantation, vol. 62, no. 7, pp. 920-926, 1996.

[6] Y. Nakata, M. Yoshibayashi, T. Yonemura et al., "Tacrolimus and myocardial hypertrophy," Transplantation, vol. 69, no. 9, pp. 1960-1962, 2000.

[7] J. M. Morales, A. Andres, M. Rengel, and J. L. Rodicio, "Influence of cyclosporin, tacrolimus and rapamycin on renal function and arterial hypertension after renal transplantation," Nephrology Dialysis Transplantation, vol. 16, supplement 1, pp. 121-124, 2001.

[8] R. Higgins, K. Ramaiyan, T. Dasgupta et al., "Hyponatraemia and hyperkalaemia are more frequent in renal transplant recipients treated with tacrolimus than with cyclosporin. Further evidence for differences between cyclosporin and tacrolimus nephrotoxicities," Nephrology Dialysis Transplantation, vol. 19, no. 2, pp. 444-450, 2004.

[9] R. Y. Chenhsu, C. C. Loong, M. H. Chou, M. F. Lin, and WU. C. Yang, "Renal allograft dysfunction associated with rifampin-tacrolimus interaction," Annals of Pharmacotherapy, vol. 34, no. 1, pp. 27-31, 2000.

[10] F. Ruschitzka, P. J. Meier, M. Turina, T. F. Lüscher, and G. Noll, "Acute heart transplant rejection due to Saint John's wort," Lancet, vol. 355, no. 9203, pp. 548-549, 2000.

[11] T. Saeki, K. Ueda, Y. Tanigawara, R. Hori, and T. Komano, "Human P-glycoprotein transports cyclosporin A and FK506," Journal of Biological Chemistry, vol. 268, no. 9, pp. 6077-6080, 1993.

[12] J. N. Roy, A. Barama, C. Poirier, B. Vinet, and M. Roger, "Cyp3A4, Cyp3A5, and MDR-1 genetic influences on tacrolimus pharmacokinetics in renal transplant recipients," Pharmacogenetics and Genomics, vol. 16, no. 9, pp. 659-665, 2006.

[13] K. N. Utecht, J. J. Hiles, and J. Kolesar, "Effects of genetic polymorphisms on the pharmacokinetics of calcineurin 
inhibitors," American Journal of Health-System Pharmacy, vol. 63, no. 23, pp. 2340-2348, 2006.

[14] G. K. Dresser and D. G. Bailey, "The effects of fruit juices on drug disposition: a new model for drug interactions," European Journal of Clinical Investigation, vol. 33, no. 2, pp. 10-16, 2003.

[15] K. Egashira, E. Fukuda, T. Onga et al., "Pomelo-induced increase in the blood level of tacrolimus in a renal transplant patient," Transplantation, vol. 75, no. 7, p. 1057, 2003.

[16] K. Egashira, H. Ohtani, S. Itoh et al., "Inhibitory effects of pomelo on the metabolism of tacrolimus and the activities of CYP3A4 and P-glycoprotein," Drug Metabolism and Disposition, vol. 32, no. 8, pp. 828-833, 2004.

[17] S. Fukatsu, M. Fukudo, S. Masuda et al., "Delayed effect of grapefruit juice on pharmacokinetics and pharmacodynamics of tacrolimus in a living-donor liver transplant recipient," Drug metabolism and pharmacokinetics, vol. 21, no. 2, pp. 122125, 2006.

[18] C. Liu, Y. F. Shang, X. F. Zhang et al., "Co-administration of grapefruit juice increases bioavailability of tacrolimus in liver transplant patients: a prospective study," European Journal of Clinical Pharmacology, vol. 65, no. 9, pp. 881-885, 2009.

[19] Y. C. Hou, S. L. Hsiu, C. W. Tsao, Y. H. Wang, and P. D. L. Chao, "Acute intoxication of cyclosporin caused by coadministration of decoctions of the fruits of Citrus aurantium and the pericarps of Citrus grandis," Planta Medica, vol. 66, no. 7, pp. 653-655, 2000.

[20] M. J. Wang, Y. C. Hou, S. Y. Tsai, S. L. Hsiu, K. C. Wen, and P. D. L. Chao, "Effects of coadministrations of unripe fruits and ripe peels of Citrus aurantium on cyclosporine pharmacokinetics in rats," Journal of Food and Drug Analysis, vol. 15, no. 3, pp. 265-270, 2007.

[21] T. Mosmann, "Rapid colorimetric assay for cellular growth and survival: application to proliferation and cytotoxicity assays," Journal of Immunological Methods, vol. 65, no. 1-2, pp. 55-63, 1983.

[22] J. X. Jia and K. M. Wasan, "Effects of monoglycerides on rhodamine 123 accumulation, estradiol 17 beta-D-glucuronide bidirectional transport and MRP2 protein expression within Caco-2 cells," Journal of Pharmacy \& Pharmaceutical Sciences, vol. 11, no. 3, pp. 45-62, 2008.

[23] C. Bistrup, F. T. Nielsen, U. E. Jeppesen, and H. Dieperink, "Effect of grapefruit juice on Sandimmum Neoral ${ }^{\circledR}$ absorption among stable renal allograft recipients," Nephrology Dialysis Transplantation, vol. 16, no. 2, pp. 373-377, 2001.

[24] U. Christians, W. Jacobsen, L. Z. Benet, and A. Lampen, "Mechanisms of clinically relevant drug interactions associated with tacrolimus," Clinical Pharmacokinetics, vol. 41, no. 11, pp. 813-851, 2002.

[25] M. Saito, M. Hirata-Koizumi, M. Matsumoto, T. Urano, and R. Hasegawa, "Undesirable effects of citrus juice on the pharmacokinetics of drugs: focus on recent studies," Drug Safety, vol. 28, no. 8, pp. 677-694, 2005.

[26] D. J. Edwards, F. H. Bellevue III, and P. M. Woster, "Identification of 6',7'-dihydroxybergamottin, a cytochrome P450 inhibitor, in grapefruit juice," Drug Metabolism and Disposition, vol. 24, no. 12, pp. 1287-1290, 1996.

[27] K. He, K. R. Iyer, R. N. Hayes, M. W. Sinz, T. F. Woolf, and P. F. Hollenberg, "Inactivation of cytochrome P450 3A4 by bergamottin, a component of grapefruit juice," Chemical Research in Toxicology, vol. 11, no. 4, pp. 252-259, 1998.

[28] G. Lian-Qing, K. Fukuda, T. Ohta, and Y. Yamazoe, "Role of furanocoumarin derivatives on grapefruit juice-mediated inhibition of human CYP3A activity," Drug Metabolism and Disposition, vol. 28, no. 7, pp. 766-771, 2000.

[29] A. Ohnishi, H. Matsuo, S. Yamada et al., "Effect of furanocoumarin derivatives in grapefruit juice on the uptake of vinblastine by Caco-2 cells and on the activity of cytochrome P450 3A4," British Journal of Pharmacology, vol. 130, no. 6, pp. 1369-1377, 2000.

[30] W. Tassaneeyakul, L.-Q. Guo, K. Fukuda, T. Ohta, and Y. Yamazoe, "Inhibition selectivity of grapefruit juice components on human cytochromes P450," Archives of Biochemistry and Biophysics, vol. 378, no. 2, pp. 356-363, 2000.

[31] P.-C. Ho, D. J. Saville, and S. Wanwimolruk, "Inhibition of human CYP3A4 activity by grapefruit flavonoids, furanocoumarins and related compounds," Journal of Pharmacy and Pharmaceutical Sciences, vol. 4, no. 3, pp. 217-227, 2001.

[32] Y. Honda, F. Ushigome, N. Koyabu et al., "Effects of grapefruit juice and orange juice components on P-glycoprotein- and MRP2-mediated drug efflux," British Journal of Pharmacology, vol. 143, no. 7, pp. 856-864, 2004.

[33] M. F. Paine, W. W. Widmer, H. L. Hart et al., "A furanocoumarin-free grapefruit juice establishes furanocoumarins as the mediators of the grapefruit juice-felodipine interaction," American Journal of Clinical Nutrition, vol. 83, no. 5, pp. 10971105, 2006.

[34] K. S. Lown, D. G. Bailey, R. J. Fontana et al., "Grapefruit juice increases felodipine oral availability in humans by decreasing intestinal CYP3A protein expression," Journal of Clinical Investigation, vol. 99, no. 10, pp. 2545-2553, 1997.

[35] P. Schmiedlin-Ren, D. J. Edwards, M. E. Fitzsimmons et al., "Mechanisms of enhanced oral availability of CYP3A4 substrates by grapefruit constituents: decreased enterocyte CYP3A4 concentration and mechanism-based inactivation by furanocoumarins," Drug Metabolism and Disposition, vol. 25, no. 11, pp. 1228-1233, 1997.

[36] G. C. Kane and J. J. Lipsky, "Drug-grapefruit juice interactions," Mayo Clinic Proceedings, vol. 75, no. 9, pp. 933-942, 2000.

[37] L. Q. Guo, M. Taniguchi, Q. Y. Chen, K. Baba, and Y. Yamazoe, "Inhibitory potential of herbal medicines on human cytochrome P450-mediated oxidation: properties of Umbelliferous or Citrus crude drugs and their relative prescriptions," Japanese Journal of Pharmacology, vol. 85, no. 4, pp. 399-408, 2001. 


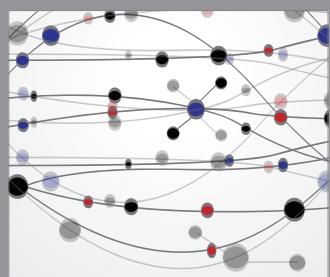

The Scientific World Journal
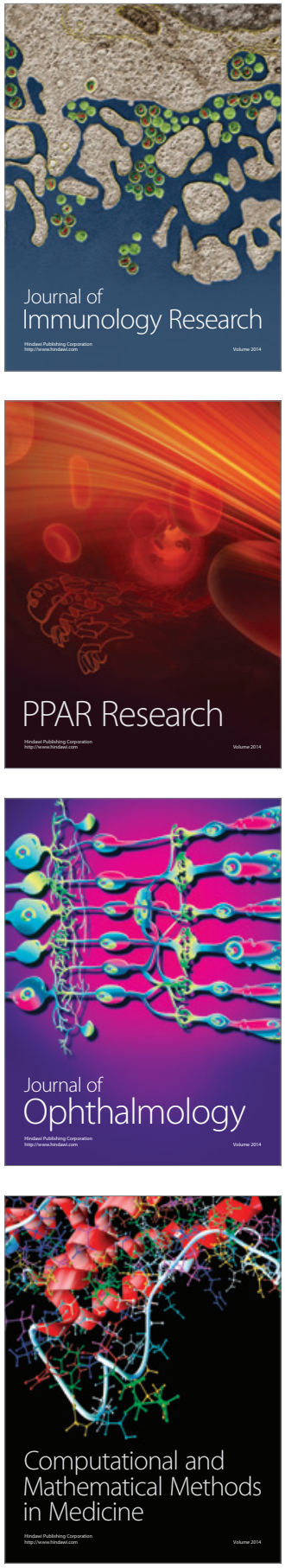

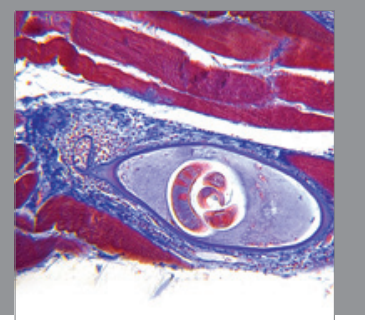

Gastroenterology

Research and Practice
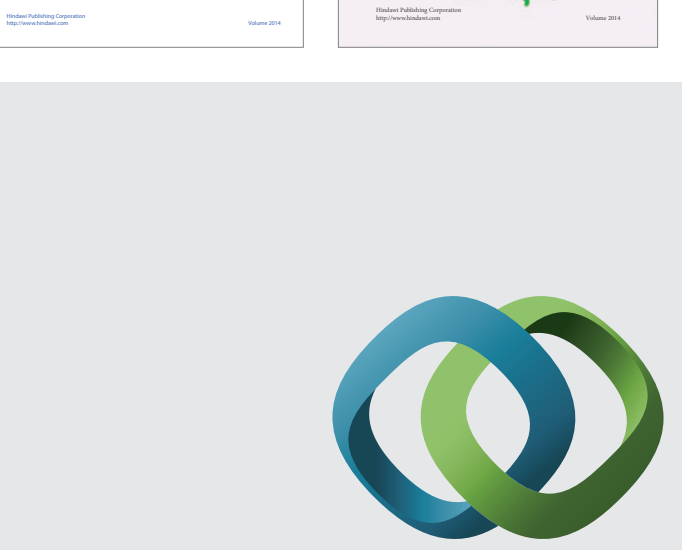

\section{Hindawi}

Submit your manuscripts at

http://www.hindawi.com
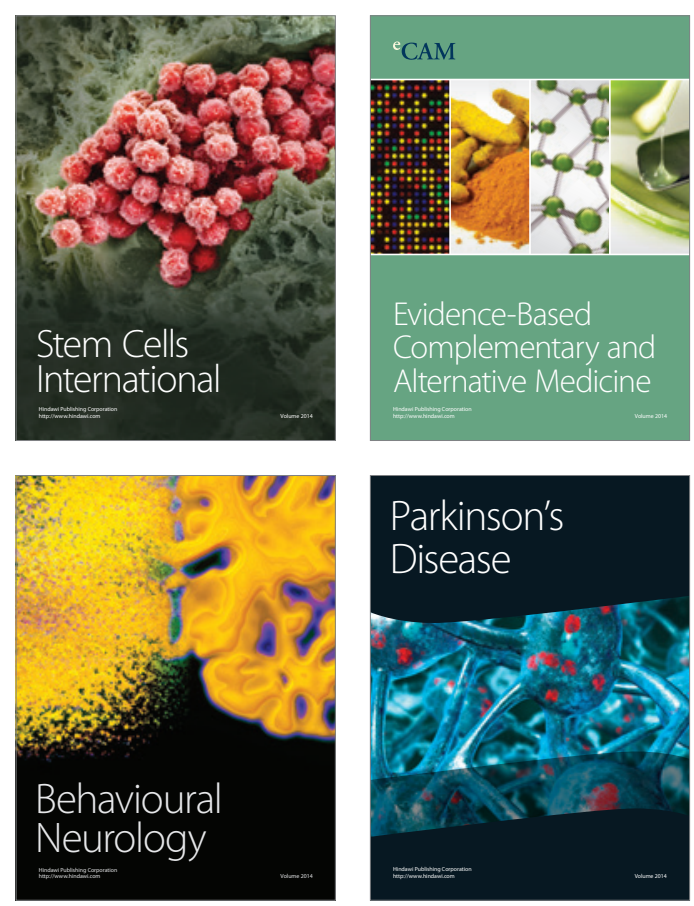

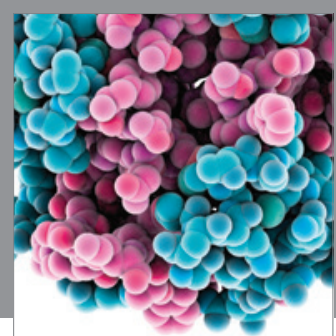

Journal of
Diabetes Research

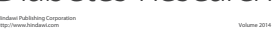

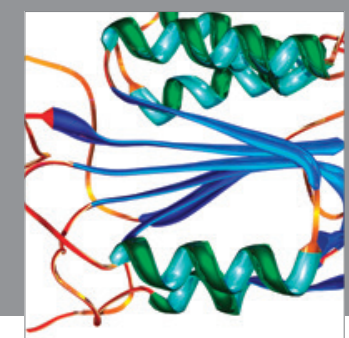

Disease Markers
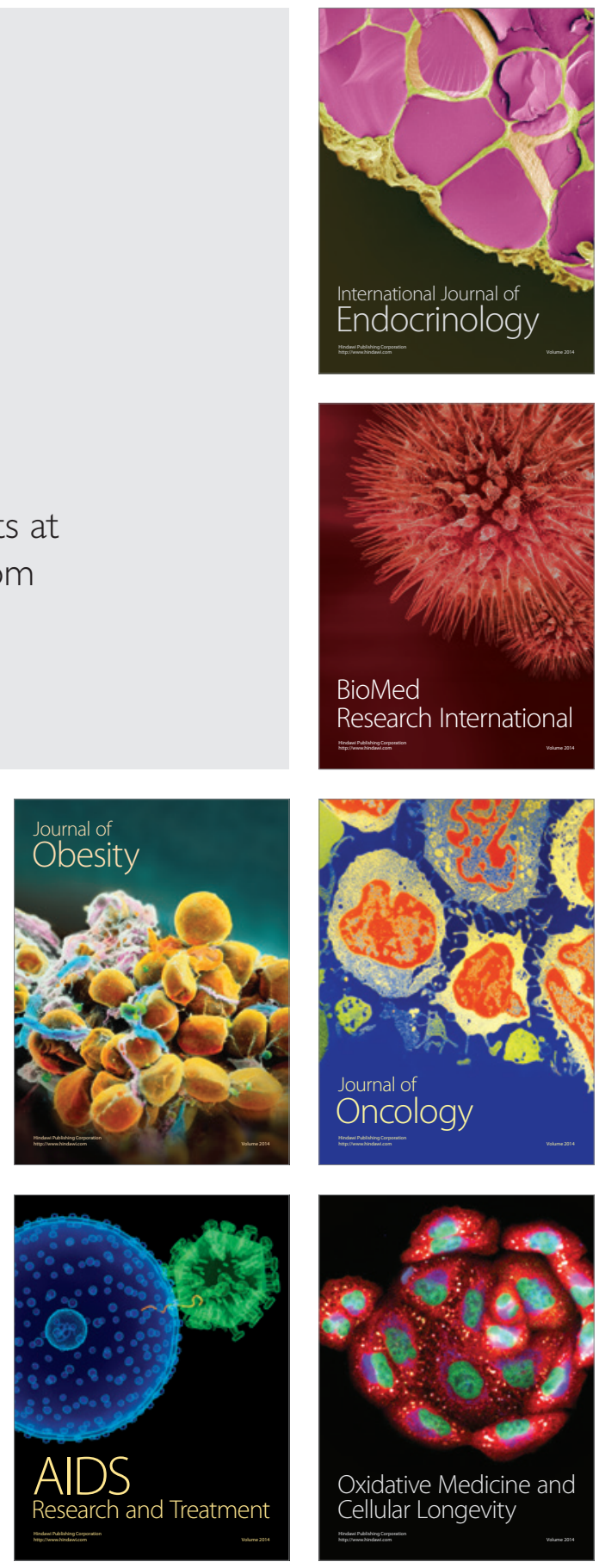